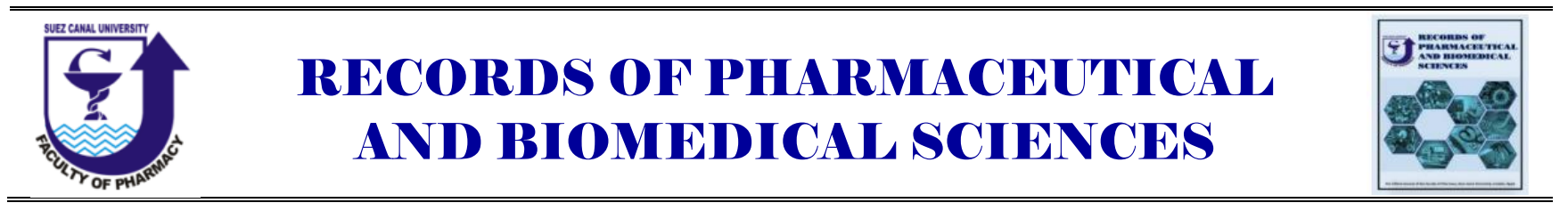

\title{
Parameter Optimization of Solid Lipid Nanoparticles Formulation
}

\author{
Alshimaa Gamal *a, Ahmed R.M. Gardouh ${ }^{b, c}$, Shadeed Gad ${ }^{b}$ \\ ${ }^{a}$ Department of Pharmaceutics and Industrial Pharmacy, Faculty of Pharmacy, Sinai University, \\ Ismailia, Egypt. ${ }^{b}$ Department of Pharmaceutics and Industrial Pharmacy, Faculty of Pharmacy, Suez \\ Canal University, Ismailia, Egypt, ${ }^{c}$ Department of Pharmaceutics and Industrial Pharmacy, Faculty of \\ Pharmacy, Heliopolis University for Sustainable Development, Cairo, Egypt.
}

Received on: 26. 01. 2020

Revised on: 05. 04. 2020

Accepted on: 08. 04. 2020

Correspondence Author:

Tel: +201285313986

E-mail address:

Alshimaa.gamal@su.edu.eg

\begin{abstract}
Solid lipid nanoparticles (SLN) are colloidal lipid carriers, which used for improving the bioavailability of lipophilic and hydrophilic drugs. SLN are composed of biodegradable lipids such as hard fats, highly purified triglycerides, monoglycerides, complex glyceride mixtures or waxes which are solid at room temperature. In the present study, different formulation variables, including the type and quantity of lipid and surfactant were optimized for the preparation of SLN. SLN were prepared by a modified micro emulsion-based method and their particle size was evaluated. Two types of surfactants Tween 80 and Poloxamer 188 were used. Also, the monoglyceride (Glyceryl monostearate) and wax (Cetyl palmitate) were used as lipid. The optimum SLN formulae were at lipid concentration $5 \%$. The optimum surfactant concentration was $6 \%$. SLN with a surfactant Tween 80 were smaller in size than Poloxamer 188 SLN. Glyceryl monostearate formed a smaller size SLN than Cetyl palmitate. Increasing Glyceryl monostearate concentration in the lipid ratio resulted in the formation of small size SLN.
\end{abstract}

Key words: solid lipid nanoparticles; modified micro emulsion-based method; high shear homogenization; Tween 80; Poloxamer 188; Glyceryl monostearate; Cetyl palmitate

\section{Introduction}

Solid lipid nanoparticles (SLN) are colloidal lipid carriers, which are composed of biodegradable lipids such as hard fats, highly purified triglycerides, monoglycerides, complex glyceride mixtures or waxes which are solid at room temperature. Unlike liposomes and emulsions, the particle-matrix of SLN is composed of solid lipids (Mulla, Hiremath et al. 2012).
There are many advantages of SLN such as the possibility of controlled drug release and targeting, the ability of incorporation of lipophilic and hydrophilic drugs, good biocompatibility and low biotoxicity, avoiding the use of organic solvents in the production cycle and easy large scale production. Oral administration of SLN could increase the lymphatic transport of drugs, which decreased first-pass hepatic metabolism and increased oral bioavailability of the drug 
(Mulla, Hiremath et al. 2012, Ebrahimi, Javadzadeh et al. 2015). Various methods were available for the production of SLN such as hot and cold highpressure homogenization, high shear homogenization and ultrasonication, solvent emulsification/evaporation process, and micro emulsion-based technique. The principle of this latter method was to disperse a warm oil-in-water microemulsion into a large amount of cold water forming SLN. So, this method was easily processed at the laboratory scale as a microemulsion, the key intermediate product of this preparation, would be formed spontaneously (Boonme, Souto et al. 2013). But the removal process of excess water was difficult so that some modifications applied to the method helped to decrease the dispersion ratio to 1:2 which improved the stability of the prepared SLNs (Aboutaleb, Noori et al. 2012). This study aimed to optimize different formulation variables, including the type and quantity of surfactant for the preparation of SLNs. Also, study the effect of using lipids with different molecular structures and different concentrations on the particle size of SLN.

\section{Experimental}

\subsection{Materials}

Cetyl palmitate was kindly provided by Gattefosee, Saint-Priest, France. Glyceryl monostearate was purchased from Alpha Chemika, Mumbai, India. Poloxamer 188 was purchased from Caisson laboratories, Utah, United States. Tween 80 was purchased from Elnasr pharmaceutical chemicals, Al Qalyubia, Egypt.

Distilled water and freshly prepared reverse osmosis (RO) water were used in all experiments. All chemicals were used directly as received without further purification.

\subsection{Methods}

\subsubsection{Preparation of solid lipid nanoparticles}

SLN were prepared by a modified micro emulsionbased technique. The lipids (cetyl palmitate/Glyceryl monostearate) and the emulsifiers (Tween 80/Poloxamer 188) were molten and mixed using magnetic stirring at $80^{\circ} \mathrm{C}$ then, up to $30 \mathrm{ml}$ of $\mathrm{RO}$ water at the same temperature was added.
This coarse pre-emulsion was treated with high shear homogenization using an Ultra-Turrax homogenizer (Ultra- Turrax T - 25, IKA, Germany) at 15,000 rpm for 15 minutes, with 30 seconds intervals every two minutes; the resulting microemulsion was immediately dispersed in cold distilled water while stirring $\left(2^{\circ} \mathrm{C}\right.$ to $\left.3^{\circ} \mathrm{C}\right)$ with a ratio $1: 2$ for distilled water and pre-emulsion respectively. The principle of this latter method is to add a warm oil-in-water microemulsion into a large amount of cold water which leads to precipitation of the lipid phase forming fine particles called SLN [2, 3]. Two different surfactants (Tween 80 and Poloxamer 188) were evaluated at three different concentrations (2\%, $4 \%$ and $6 \% \mathrm{w} / \mathrm{w}$ ) and $1 \%$ as a co-surfactant. Two types of lipids (cetyl palmitate and glyceryl monostearate (GMS)) were used at three different concentrations $(1 \%, 5 \%$ and $10 \% \mathrm{w} / \mathrm{w})$ as shown in Table 1.

\subsubsection{Particle size analysis}

Particle size was measured using dynamic laser light scattering apparatus at $25^{\circ} \mathrm{C}$ (Mastersizer 2000 vers. 5.54, hydro 2000 S, Malvern Instruments Ltd., Malvern, Worcs, UK). Prior to the measurements, all samples were diluted using ultra-purified water to yield a suitable scattering intensity. DLS data were analyzed at $25^{\circ} \mathrm{C}$ using the general-purpose mode.

\section{Results and Discussion \\ 3.1. Effect of surfactant type and concentration on the particle size of SLN}

The use of nonionic surfactants increased SLN stability through their steric properties (Ebrahimi, Javadzadeh et al. 2015). As shown in Error! Reference source not found., low molecular weight surfactants gave smaller size SLN due to their ability to cover interfacial spaces more quickly, where Tween 80 (molecular weight 1310 Dalton's) formed smaller SLN than Poloxamer 188 (Avg. molecular weight 8400 Dalton's). This property was observed in a previous study, where Tween 80and phosphatidylcholine based SLN formed a smaller particle size than polyvinyl alcohol (PVA) and Polyvinyl pyrrolidone (PVP) based SLN (Ebrahimi, Javadzadeh et al. 2015). 
Rec. Pharm. Biomed. Sci. C: 4 (2), 1-7, 2020

Table 1. Composition of various SLN at different lipid concentration 1, 5 and $10 \%$ using different surfactant concentration and different lipid ratio

\begin{tabular}{|c|c|c|c|c|c|c|}
\hline \multicolumn{3}{|c|}{ SLN Formula code } & \multirow{2}{*}{$\begin{array}{l}\text { Cetyl palmitate } \\
\text { in lipid ratio }\end{array}$} & \multirow{2}{*}{$\begin{array}{c}\text { Glyceryl } \\
\text { monostearte } \\
\text { in lipid ratio }\end{array}$} & \multirow{2}{*}{$\begin{array}{l}\text { Tween } 80 \\
(w / w \%)\end{array}$} & \multirow{2}{*}{$\begin{array}{c}\text { Poloxamer } \\
188(w / w \%)\end{array}$} \\
\hline $\begin{array}{l}\text { Prepared at lipid } \\
\text { concentration } 1 \%\end{array}$ & $\begin{array}{l}\text { Prepared at lipid } \\
\text { concentration 5\% }\end{array}$ & $\begin{array}{c}\text { Prepared at lipid } \\
\text { concentration } 10 \%\end{array}$ & & & & \\
\hline FA11 & FB11 & $\mathrm{FC} 11$ & 1 & 0 & 2 & 1 \\
\hline FA12 & FB12 & $\mathrm{FC} 12$ & 1 & 0 & 4 & 1 \\
\hline FA13 & FB13 & $\mathrm{FC} 13$ & 1 & 0 & 6 & 1 \\
\hline FA14 & FB14 & $\mathrm{FC} 14$ & 1 & 0 & 1 & 2 \\
\hline FA15 & FB15 & $\mathrm{FC} 15$ & 1 & 0 & 1 & 4 \\
\hline FA16 & FB16 & FC16 & 1 & 0 & 1 & 6 \\
\hline FA21 & FB21 & $\mathrm{FC} 21$ & 0 & 1 & 2 & 1 \\
\hline FA22 & FB22 & $\mathrm{FC} 22$ & 0 & 1 & 4 & 1 \\
\hline FA23 & FB23 & $\mathrm{FC} 23$ & 0 & 1 & 6 & 1 \\
\hline FA24 & FB24 & $\mathrm{FC} 24$ & 0 & 1 & 1 & 2 \\
\hline FA25 & FB25 & $\mathrm{FC} 25$ & 0 & 1 & 1 & 4 \\
\hline FA26 & FB26 & FC26 & 0 & 1 & 1 & 6 \\
\hline FA31 & FB31 & FC31 & 1 & 1 & 2 & 1 \\
\hline FA32 & FB32 & FC32 & 1 & 1 & 4 & 1 \\
\hline FA33 & FB33 & FC33 & 1 & 1 & 6 & 1 \\
\hline FA34 & FB34 & FC34 & 1 & 1 & 1 & 2 \\
\hline FA35 & FB35 & FC35 & 1 & 1 & 1 & 4 \\
\hline FA36 & FB36 & FC36 & 1 & 1 & 1 & 6 \\
\hline FA41 & FB41 & FC41 & 1 & 2 & 2 & 1 \\
\hline FA42 & FB42 & $\mathrm{FC} 42$ & 1 & 2 & 4 & 1 \\
\hline FA43 & FB43 & FC43 & 1 & 2 & 6 & 1 \\
\hline FA44 & FB44 & FC44 & 1 & 2 & 1 & 2 \\
\hline FA45 & FB45 & $\mathrm{FC} 45$ & 1 & 2 & 1 & 4 \\
\hline
\end{tabular}




\begin{tabular}{|c|c|c|c|c|c|c|}
\hline FA46 & FB46 & FC46 & 1 & 2 & 1 & 6 \\
\hline FA51 & FB51 & FC51 & 2 & 1 & 2 & 1 \\
\hline FA52 & FB52 & FC52 & 2 & 1 & 4 & 1 \\
\hline FA53 & FB53 & FC53 & 2 & 1 & 6 & 1 \\
\hline FA54 & FB54 & FC54 & 2 & 1 & 1 & 2 \\
\hline FA55 & FB55 & FC55 & 2 & 1 & 1 & 4 \\
\hline FA56 & FB56 & FC56 & 2 & 1 & 1 & 6 \\
\hline FA61 & FB61 & FC61 & 1 & 3 & 2 & 1 \\
\hline FA62 & FB62 & FC62 & 1 & 3 & 4 & 1 \\
\hline FA63 & FB63 & FC63 & 1 & 3 & 6 & 1 \\
\hline FA64 & FB64 & FC64 & 1 & 3 & 1 & 2 \\
\hline FA65 & FB65 & FC65 & 1 & 3 & 1 & 4 \\
\hline FA66 & FB66 & FC66 & 1 & 3 & 1 & 6 \\
\hline FA71 & FB71 & FC71 & 3 & 1 & 2 & 1 \\
\hline FA72 & FB72 & $\mathrm{FC72}$ & 3 & 1 & 4 & 1 \\
\hline FA73 & FB73 & $\mathrm{FC73}$ & 3 & 1 & 6 & 1 \\
\hline FA74 & FB74 & FC74 & 3 & 1 & 1 & 2 \\
\hline FA75 & FB75 & FC75 & 3 & 1 & 1 & 4 \\
\hline FA76 & FB76 & FC76 & 3 & 1 & 1 & 6 \\
\hline
\end{tabular}

Tween 80 had a long hydrophobic tail which might initiate crystallization of the lipid matrix of nanoparticles due to its affinity to the lipid, a small portion of the surfactant molecules localized in the lipid matrix not only attached to the surface and thus promoted lipid recrystallization. So, surfactant was able to stabilize the formed droplets when they left the homogenization gap to avoid coalescence. Similar results had been obtained for SLN containing Cetyl palmitate as solid lipid and Plurol Stearique (PS), Plantacare 810 (PL) as emulsifiers (Kovacevic, Savic et al. 2011). It seemed that surfactant molecular weight and structure played an important role in the particle size of SLN.

Decrease of surfactant concentration led to an increase in the particle size because the surfactant was insufficient to cover the interface and made particles aggregation. Also, increased the surfactant concentration led to a decrease in the particle size due to the reduction in interfacial tension between the aqueous and lipid phases and form a steric barrier on the particle surface, thereby protecting the particles from coagulation(Reddy and Murthy 2005). Error! Reference source not found. showed the obtained results. At high surfactant concentrations, bridging between the nanoparticles might be formed and might cause toxicity (Pooja, Tunki et al. 2016). Therefore, two different surfactants (Tween 80 and Poloxamer 188) were evaluated at three different concentrations $(2,4$ and $6 \% \mathrm{w} / \mathrm{v})$. The optimum concentration of Poloxamer 188 was $1 \%$ of the 
total formula and up to $10 \%$ of total surfactant
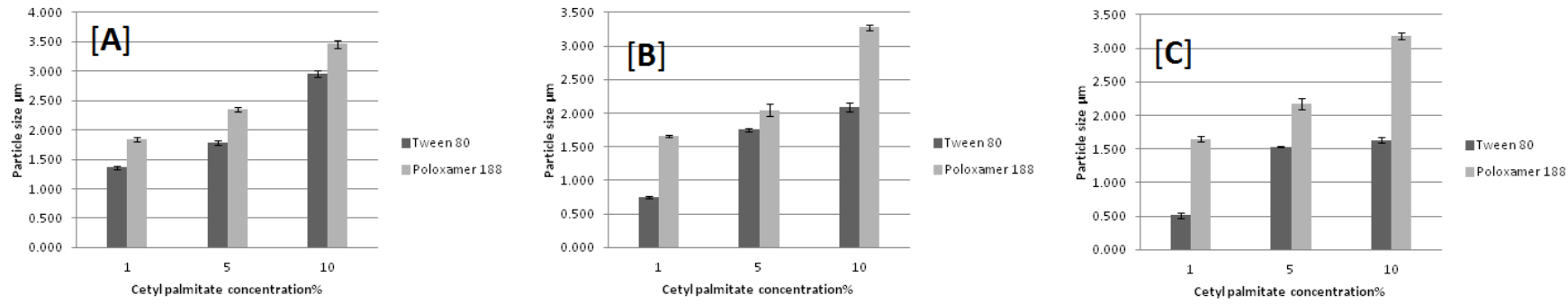

Fig. 1: Effect of surfactant type on the particle size of SLN containing Cetyl palmitate as a lipid: (A), surfactant concentration $2 \%$; (B), surfactant concentration $4 \%$; (C), surfactant concentration $6 \%$ $($ mean $\pm \mathrm{SE}, \mathrm{N}=3$ )
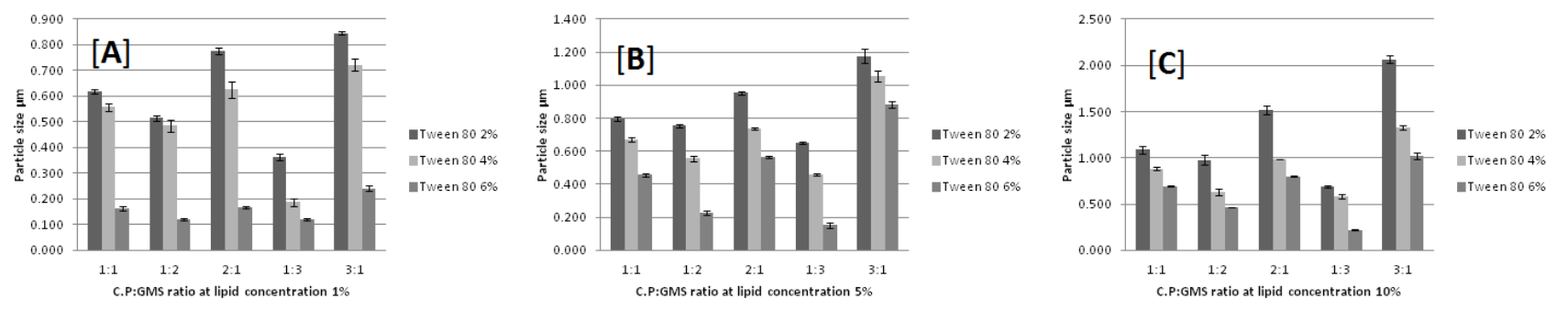

Fig. 2: Effect of Tween 80 concentration on the particle size of SLN: (A), lipid concentration 1\%; (B), lipid concentration $5 \%$; (c), lipid concentration $10 \%($ mean $\pm \mathrm{SE}, \mathrm{N}=3)$
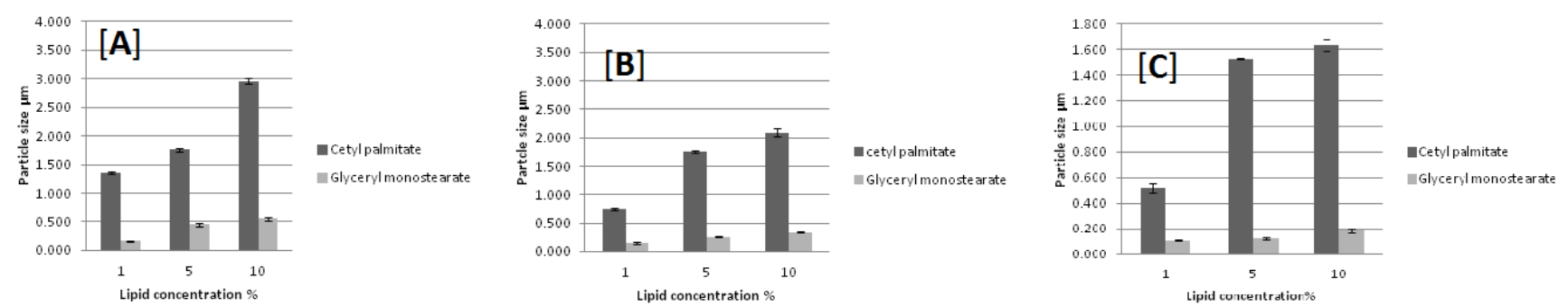

Fig. 3: Effect of lipid type and concentration on the particle size of SLN using Tween 80 as a surfactant: (A), surfactant concentration $2 \%$; (B), surfactant concentration $4 \%$; (C), surfactant concentration $6 \%$ $($ mean \pm SE, $\mathbf{N}=3$ )
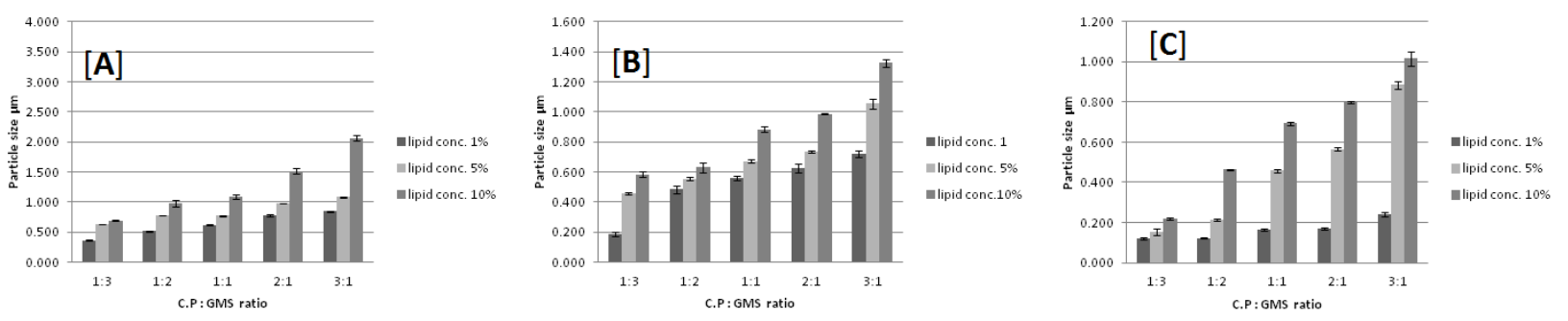

Fig. 4: Effect of increasing Cetyl palmitate concentration in lipid ratio on the particle size of SLN using Tween 80 as a surfactant: (A), surfactant concentration 2\%; (B), surfactant concentration 4\%; (C), surfactant concentration $6 \%($ mean $\pm \mathrm{SE}, \mathrm{N}=3)$ 
(Kumar, Chandrasekar et al. 2007, Aboutaleb, Noori et al. 2012). High Poloxamer concentrations (more than $1.5 \%$ ) would make a decrease in entrapment efficiency and caused toxic effects (Kumar, Chandrasekar et al. 2007). The increase in Poloxamer concentration might also increase the particle size due to the extensive dehydration of propylene oxide and ethylene oxide blocks within the Poloxamer molecule during emulsification and hot homogenization conditions, leading to reduction of steric repulsion activity (Reddy and Murthy 2005). Tween 80 at concentration $6 \%$ was optimum to give stable SLN. This result was agreed with that obtained by Aboutaleb et al. on Cetyl palmitate SLN using Tween 80 and Poloxamer 188 as surfactants for improving the antimycobacterial activity of rifampin (Aboutaleb, Noori et al. 2012).

\subsection{Effect of lipid type and concentration on the particle size of SLN}

The size of SLN was affected by the number of carbon atoms in the fatty acid chain of the lipid (Baek, Shin et al. 2012, Boonme, Souto et al. 2013). Glyceryl monostearate had a smaller number of carbon atoms on its fatty acid chain than Cetyl palmitate resulting in smaller size SLN (Error! Reference source not found.). Also, increasing the carbon chain length of fatty acid enhanced the entrapment efficiency and drug loading of SLN, because the longer chain fatty acids were more hydrophobic and would increase the accommodation of lipophilic drugs (Baek, Shin et al. 2012).

Glyceryl monostearate is a monoglyceride and it was reported that emulsifying monoglycerides might lead to a different content of water in the SLN lipid matrix which decreased the stability of SLN (Radomska-Soukharev 2007). Stability of SLN prepared with wax (Cetyl palmitate) was greater than glyceride SLN (Glyceryl monostearate) (Kumar, Chandrasekar et al. 2007).

Increase of the lipid content led to an increase in the particle size of SLN due to increase of viscosity of organic phase that resulted in slower diffusion of the organic phase in the continuous phase (Kommavarapu, Maruthapillai et al. 2015). The lipid content increased the packing density of lipid molecules in a given space which reduced the drug release(Narala and Veerabrahma 2013). Employing more than $5 \%$ of lipid led to increasing the size of SLN in micrometer range. These results were in agreement with previous studies (Aboutaleb, Noori et al. 2012, Mulla, Hiremath et al. 2012). Low lipid concentration (1 and 2\%) led to drug deposition indicating the poor encapsulation efficiency of the drug (Kumar, Chandrasekar et al. 2007).

According to the previous results, increasing Cetyl palmitate concentration in the lipid ratio resulted in a formation of large size SLN (Error! Reference source not found.) and increasing Glyceryl mono-stearate concentration in the lipid ratio resulted in the formation of small size SLN.

\section{Conclusion}

The particle size of SLN depended on the type and the concentration of the surfactant and lipid. Tween 80 gave smaller particle size SLN than Poloxamer188. Increasing the surfactant concentration led to a decrease in the particle size of SLN. Tween 80 at concentration $6 \%$ was optimum to give small size SLN and the optimum concentration of Poloxamer 188 was $1 \%$ of the total formula.

Employing 5\% of lipid was optimum and Glyceryl monostearate gave smaller size SLN than Cetyl palmitate but Cetyl palmitate enhanced the stability of SLN.

\section{References}

Aboutaleb, E., M. Noori, N. Gandomi, F. Atyabi, M. R. Fazeli, H. Jamalifar and R. Dinarvand (2012). "Improved antimycobacterial activity of rifampin using solid lipid nanoparticles." International Nano Letters 2(1): 33.

Baek, J.-S., S.-C. Shin and C.-W. Cho (2012). "Effect of lipid on physicochemical properties of solid lipid nanoparticle of paclitaxel." Journal of Pharmaceutical Investigation 42(5): 279-283.

Boonme, P., E. B. Souto, N. Wuttisantikul, T. Jongjit and W. Pichayakorn (2013). "Influence of lipids on the properties of solid lipid nanoparticles from microemulsion technique." European Journal of Lipid Science and Technology 115(7): 820-824.

Ebrahimi, H. A., Y. Javadzadeh, M. Hamidi and M. B. Jalali (2015). "Repaglinide-loaded solid lipid nanoparticles: effect of using different surfactants/stabilizers on physicochemical properties of nanoparticles." DARU Journal of Pharmaceutical Sciences 23(1): 46. 
Kommavarapu, P., A. Maruthapillai and K. Palanisamy (2015). "Preparation, characterization and evaluation of elvitegravir-loaded solid lipid nanoparticles for enhanced solubility and dissolution rate." Tropical Journal of Pharmaceutical Research 14(9): 1549-1556.

Kovacevic, A., S. Savic, G. Vuleta, R. Müller and C. Keck (2011). "Polyhydroxy surfactants for the formulation of lipid nanoparticles (SLN and NLC): effects on size, physical stability and particle matrix structure." International journal of pharmaceutics 406(1-2): 163-172.

Kumar, V. V., D. Chandrasekar, S. Ramakrishna, V. Kishan, Y. M. Rao and P. V. Diwan (2007). "Development and evaluation of nitrendipine loaded solid lipid nanoparticles: influence of wax and glyceride lipids on plasma pharmacokinetics." International journal of pharmaceutics 335(1-2): 167-175.

Mulla, J. A. S., S. P. Hiremath and N. K. Sharma (2012). "Repaglinide loaded solid lipid nanoparticles: design and characterization." RGUHS J Pharm Sci 2(4): 41-49.

Narala, A. and K. Veerabrahma (2013). "Preparation, characterization and evaluation of quetiapine fumarate solid lipid nanoparticles to improve the oral bioavailability." Journal of pharmaceutics 2013.

Pooja, D., L. Tunki, H. Kulhari, B. B. Reddy and R. Sistla (2016). "Optimization of solid lipid nanoparticles prepared by a single emulsificationsolvent evaporation method." Data in brief 6: 1519.

Radomska-Soukharev, A. (2007). "Stability of lipid excipients in solid lipid nanoparticles." Advanced drug delivery reviews 59(6): 411-418.

Reddy, L. H. and R. Murthy (2005). "Etoposideloaded nanoparticles made from glyceride lipids: formulation, characterization, in vitro drug release, and stability evaluation." AAPs PharmSciTech 6(2): E158-E166. 